Voix et Images

voixetimages

\title{
Bibliographie de Suzanne Lamy
}

\section{Pascale Noizet}

Volume 13, numéro 1 (37), automne 1987

\section{Suzanne Lamy}

URI : https://id.erudit.org/iderudit/200686ar

DOI : https://doi.org/10.7202/200686ar

Aller au sommaire du numéro

Éditeur(s)

Université du Québec à Montréal

ISSN

0318-9201 (imprimé)

1705-933X (numérique)

Découvrir la revue

Citer ce document

Noizet, P. (1987). Bibliographie de Suzanne Lamy. Voix et Images, 13(1), 70-80. https://doi.org/10.7202/200686ar

Ce document est protégé par la loi sur le droit d'auteur. L'utilisation des services d'Érudit (y compris la reproduction) est assujettie à sa politique d'utilisation que vous pouvez consulter en ligne.

https://apropos.erudit.org/fr/usagers/politique-dutilisation/
Cet article est diffusé et préservé par Érudit.

Érudit est un consortium interuniversitaire sans but lucratif composé de l’Université de Montréal, l’Université Laval et l'Université du Québec à Montréal. Il a pour mission la promotion et la valorisation de la recherche. https://www.erudit.org/fr/ 


\title{
Bibliographie de Suzanne Lamy
}

\author{
par Pascale Noizet, Université du Québec à Montréal
}

\section{OEUVRES}

La Renaissance des métiers d'art au Canada français (en collaboration avec Laurent Lamy), Québec, Ministère des Affaires culturelles, 1967, 84 p.

André Breton, Hermétisme et poésie dans Arcane 17, Montréal, Presses de l'Université de Montréal, 1977, 265 p.

d'elles, Montréal, l'Hexagone, 1979, 110 p.

Marguerite Duras à Montréal (en collaboration avec André Roy), Textes réunis et présentés par S. Lamy et A. Roy, Montréal, Éditions Spirale, 1981, 175 p. [Paris, Editions Solin, 1984].

Féminité, subversion, écriture (en collaboration avec Irène Pagès), Textes réunis et présentés par S. Lamy et I. Pagès, Montréal, Éditions du Remue-ménage, 1983,286 p.

Quand je lis je m'invente, Montréal, l'Hexagone, 1984, 111 p.

La Convention (récit), Montréal/Paris, VLB éditeur/le Castor astral, 1985, 95 p.

\section{CHAPITRES DE VOLUMES}

«Louise Maheux-Forcier, Un arbre chargé d'oiseaux, téléthêâtre précédé du journal de la maison d'Irène», in Livres et auteurs québécois 1976, Québec, Presses de l'Université Laval, 1977, p. 184-186.

«Multiple» suivi de «Nietzsche, Freud et les femmes. Entretien avec Luce Irigaray» (en collaboration avec André Roy), in Luce Irigaray, le Corps-à-corps avec la mère, Montréal, Éditions de la Pleine Lune, 1981, p. 37-72.

«Au jeu du désir et de la mort: Journal d'une femme soumise de Mara et l'Homme assis dans le couloir de M. Duras», in Marcelle Brisson, Éros au pluriel, Montréal, Hurtubise HMH, collection «Brèches», 1984, p. 63-93. 


\section{DOSSIER 71}

\section{ARTICLES DE PÉRIODIQUES ET DE JOURNAUX}

\section{Chatelaine}

\section{Chronique «Les Arts et les innovatrices*}

«Martine Epoque-Poulin et la danse moderne», vol. 12, no 9, septembre 1971, p. 10.

«le Cinéma d'animation», vol. 12, no 10 , octobre 1971, p. 6.

«Inventer des sons nouveaux. Micheline Coulombe Saint-Marcoux», vol. 12, no 11 , novembre 1971, p. 14.

«l'Exposition du groupe automatiste. Marcelle Ferron», vol. 12, no 12, décembre 1971, p. 12.

«la Sculpture en mouvement. Luce Dupuy», vol. 13, no 1, janvier 1972, p. 16.

«la Poésie accessible à tous. Michèle Rossignol», vol. 13, no 2, février 1972, p. 22.

«Une oeuvre où la femme est libre. Claire Martin», vol. 13, no 3, mars 1972, p. 12 .

«Nouvelle galerie d'art. Jeanne Renaud», vol. 13, no 4, avril 1972, p. 14.

"Un film témoin d'une génération. Michèle Lalonde», vol. 13, no 5, mai 1972, p. 10.

«Oeuvres d'art pour tous. Suzèle Carle», vol. 13, no 6, juin 1972, p. 10.

«la Comédie musicale québécoise. Marjolaine Hébert», vol. 13, no 7, juillet 1972, p. 17.

«Des films sur la femme aujourd'hui. Anne-Claire Poirier», vol. 13, no 8, août 1972 , p. 10.

«Du thêatre pour les jeunes. Françoise Graton», vol. 13, no 9, septembre 1972, p. 10 .

"Un thêâtre aussi violent que tendre. Jacqueline Barrette», vol. 13, no 10, octobre 1972, p. 16.

«Une galerie tenue par des artistes. Lise Bissonnette», vol. 13, no 11, novembre 1972 , p. 14 .

«Pour Noël 72, une charlotte «électrique». Nicole Leblanc», vol. 13, no 12, décembre 1972, p. 14. 
«Et l'ogre se transforma en souris... Nicole Lapointe», vol. 14, no 1, janvier 1973, p. 14.

«Langage son et images. Henriette Major», vol. 14, no 2, février 1973, p. 14.

«Figure de révolte et de dignité. La Sagouine d'Antonine Maillet», vol. 14, no 3, mars 1973, p. 10.

\section{Chronique *l'Art du tempsr}

«Chantal Belhumeur... Avec un nom pareil!», vol. 14, no 4, avril 1973, p. 4850.

«le Musée des Beaux arts, c'est votre affaire», vol. 14, no 5, mai 1973, p. 16.

«le T.P.Q. en scène depuis 10 ans», vol. 14, no 6, juin 1973, p. 82.

«Danièle Suissa et la Sagouine pourront-elles s'entendre?», vol. 14, no 7 , juillet 1973 , p. 17.

«Ils s'voient tous déjà en haut de l'affiche...», vol. 14, no 8, août 1973, p. 9.

«Seuls mais avec d'autres», vol. 14, no 9, septembre 1973, p. 8.

«A Québec c'est tout un festival», vol. 14 , no 10 , octobre 1973, p. 31.

«'Oeil féroce d'un homme doux», vol. 14, no 10 (sic), novembre 1973, p. 24.

«Radio-Québec UHF? Connais pas», vol. 14, no 12, décembre 1973, p. 17.

Spirale

«Pouvoir du discours et discours du pouvoir. Leçon de R. Barthes. Hélène Cixous. Trois conférences à Montréal», no 5, janvier 1980, p. 9.

«le Féminisme passé, présent et futur. Les Femmes et leurs maîtres de M.A. Macciocchi», no 6, février 1980 , p. 5-6.

«On ne naît pas minoritaire, on le devient. Minorités dans la pensée, Collectif», no 7 , mars 1980 , p. 6-7.

«Médecine, code ou bouffe heureuse? La Malbouffe de S. et J. de Rosnay. Le Mangeur du XIXe siècle de J.-P. Aron. Le Corps à corps culinaire de N. Châtelet», no 8 , avril 1980, p. 6.

«le Pouvoir des noms propres. Quand prime le spirituel de S. de Beauvoir. Ti Jean l'horizon de S. Schwarz-Bart. Héloïse d'A. Hébert», no 10, juin 1980, p. 1 et 4.

«l'Appel du désir. Le Navire night et l'Homme assis dans le couloir de M. Duras», no 11 , septembre 1980 , p. 1 et 4. 
«les Femmes et la critique. Comment commencer?», no 11, septembre 1980, p. 8. «Nietzsche, Marx, Freud et les femmes. Entretien avec Luce Irigaray» (en collaboration avec André Roy), no 12, octobre 1980, p. 3.

«Tribulations et avatars culturels. La Culture pour vivre de J. Rigaud», no 12 , octobre 1980 , p. 4.

«l'Éternel maternel en question. L'Amour en plus d'É. Badinter», no 13, novembre 1980 , p. 13.

«Blanchot, l'écriture et les femmes. Entretien avec Françoise Collin», no 15, janvier 1981 , p. 4.

«le Sens de 1'histoire. Femmes et Russie, collectif '1'Almanach'», no 16, février 1981, p. 4.

«De l'utilisation du féminisme. Fanny de E. Jong», no 17, mars 1981, p. 3-4.

«Michèle Causse: Face au déni, l'encontre. Entretien», no 18, avril 1981, p. 6.

«Une pastille à odeur d'encens. Prélude à une naissance d'A. et J.-M. Delacroix», no 19 , mai 1981, p. 4.

«Marguerite Duras à Montréal», no 20, juin 1981, p. 11.

«Thérapie à coups d'épingles. Petite soeur née... prépare suicide d'O. Dhavernas», no 20 , juin 1981, p. 9.

«Hilarant et merveilleux Dada. Coeur à gaz de T. Tzara», no 20, juin 1981, p. 19.

«Rechercher ce qu'on ne sait pas encore. Entretien avec Viviane Forrester», no 21, septembre 1981, p. 24 et 17.

«Clérambault, vous connaissez? La Passion des étoffes chez un neuro-psychiatre, G.G. de Clérambault, Collectif», no 22, février 1982, p. 16.

«Anna, Soror de M. Yourcenar et le Choc amoureux de F. Alberoni», no 22, février 1982, p. 11.

«Ligne de crête, ligne de coeur. Fragments d'art total de P. Chamberland», no 23, mars 1982, p. 14.

«le Corps et l'éthique. Entretien avec Chantal Chawaf», no 24, avril 1982, p. 6.

«Un saut (manqué) dans la mêlée. L'Écriture-femme de B. Didier», no 24, avril 1982 , p. 8.

«la Parole peut tuer. Entretien avec Bernard de Fréminville», no 25, mai 1982, p. 10. 
"Laissée en souffrance. Les Allées cavalières de V. Forrester», no 25, mai 1982, p. 7.

«Une histoire de mort et d'amour. La Cérémonie des adieux suivi de Entretiens avec J.-P. Sartre de S. de Beauvoirs (en collaboration avec Jeanne Goldin), no 26 , juin 1982 , p. 6 .

«Comment se perd la virginité. La Première fois ou le roman de la virginité perdue à travers les siècles et les continents, en collaboration», no 27, septembre 1982, p. 4.

«Voix de l'intérieur. L'Excès-usine de L. Kaplan», no 27, septembre 1982, p. 13.

«De la loi de la lettre et de l'esprit. Grammaire typographique de A. Ramat et l'Oreille de l'autre, textes et débats avec Derrida», no 28, octobre 1982, p. 2-3.

«Un bon tour d'Umberto Eco. Le Nom de la rose d'U. Eco», no 28, octobre 1982, p. 11 .

«le Roman de l'irresponsabilité. Les Fous de Bassan d'A. Hébert», no 29, novembre 1982, p. 3 et 2.

«Excellent, MAIS. Manuel bibliographique des études littéraires de B. Beugnot et J.-M. Moureaux», no 30, décembre 1982, p. 7.

«Marre de leurs acrobaties, enfin une écriture. Marie Tiefenthaler de M. Cerf. Une baraque rouge et moche comme tout, à Venice, Amérique de M.-G. LandesFuss, Monsieur Songe de R. Pinget», no 31, février 1983, p. 6.

«Québec 10/10, Biblio, Collection «Balises», Aube de J. Hogue», no 32, mars 1983, p. 5.

«Ravie de n'être pas respectable. Le Respect des femmes de S. Kofman», no 32, mars 1983, p. 11.

«Quand le réel résiste à la coïncidence», no 33, avril 1983, p. 8.

«Délices ou fadeur de l'eau claire. Les Pays étrangers de J. Ethier-Blais», no 33, avril 1983 , p. 5 .

«Sur le parcours détourné de soi. La Bête dans la jungle de J. Lord», no 34, mai 1983, p. 5.

«Le Rapport Hite sur les hommes de S. Hite», no 35, juin 1983, p. 13.

«Dictionnaire des symboles de J. Chevalier et A. Gheerbrant», no 35, juin 1983, p. 15 .

«Qui analyse qui? Van Gogh ou l'enterrement dans les blés de V. Forrester», no 35 , juin 1983 , p. 8-9. 
«le Chat dans tous ses états de J.-L. Hue», no 36, septembre 1983, p. 10.

«Dires (vol. I, no 1)» no 36 , septembre 1983, p. 8.

«Pas assez ou trop de méthode. La Littérature intime du Québec de F. Van RoeyRoux», no 36, septembre 1983, p. 4.

«Vlasta (no 1, printemps 1983)», no 37, octobre 1983, p. 7.

«Philosopher sur le champ. Les Carnets de la drôle de guerre de J.-P. Sartre», no 37, octobre 1983, p. 11.

«M.D. de Y. Andréa et Irpinia de F. Caccia», no 38, novembre 1983, p. 6.

«Les Québécois en France. Magda la rivière de F. Dépatie», no 38, novembre 1983 , p. 3.

«Un texte aseptique. Comme des mannequins de R. Lapierre», no 39, décembre 1983, p. 11.

«Quand l'émotion est intellectuelle. Texte. Montaigne en mouvement de J. Starobinski. La Table de F. Ponge», no 40, février 1984, p. 7-8.

«Retrouvailles ou premières lectures. L'Érotisme des années folles: le con d'Irène et Roger ou les à-côtés de l'ombrelle. Lectures érotiques de J.-J. Pauvert. L'Opoponax de M. Wittig», no 41, mars 1984, p. 12.

«le Texte - le sens - l'invention de la vie. Le Surréalisme et le roman de J. Chénieux-Gendron», no 42 , avril 1984 , p. 16.

"Qu'en est-il de l'invention. Revue Liberté (décembre 1983), Québec français (mars 1984), la Machine littéraire d'I. Calvino», no 43, mai 1984, p. 7.

«le Texte-iceberg. Journal intime de N. Brossard», no 44, juin 1984, p. 9.

«Comment, pourquoi parler de soi? Journal ordinaire de Mara. La Vie arrachée de M. Mailhot», no 45, septembre 1984 , p. 7.

«Dictionnaire de la bêtise et des erreurs de jugement de G. Bechtel et J.-C. Carrièrem, no 46, octobre 1984, p. 12.

«Diversité de tons et de trajets. Corps écrit (no 8). Gradiva (nos 1 et 2)», no 46 ,octobre 1984 , p. 5 .

«Duras encore. L'Amant, Théâtre III.», no 47, novembre 1984, p. 4.

«l'Ours de M. Engel», no 47, novembre 1984, p. 2.

«De la jouissance à consommer. Les Contes et leurs fantasmes de J. BelleminNoël», no 47, novembre 1984, p. 7. 
«Au noeud du privé et du politique. Entretien avec Robert Castel», no 48, décembre 1984, p. 15-16.

«le Nouveau commerce (Cahier 58 - printemps 1984)», no 48, décembre 1984, p. 6.

«les Jeux de massacre, vous aimez? Quelle douleur de M. Larouche-Thibault», no 48 , décembre 1984 , p. 10.

«Découverte et discrétion assurées. Voyage en Irlande avec un parapluie de L. Gauthier», no 49 , févriet 1985 , p. 5.

«Tirer ou délier la langue. Dictionnaire des difficultés de la langue française au Canada de G. Dagenais. Le Littré en 10/18. Petit Larousse illustré», no 50, mars 1985, p. 10.

«S comme Souffle, comme Souffrir. Le Souffle coupé de F.-B. Michel», no 51, avril 1985 , p. 11.

"Adrien le bien-nommé. Adrien de peine et misère d'Y. Lacroix», no 52, mai 1985 , p. 4.

«Françoise Collin: la diversité des pratiques», no 53, juin 1985, p. 14.

«es Cahiers du Grif», no 53, juin 1985, p. 10.

«Surréalisme et travail du temps. Lettres à Gala de P. Éluard. Le Surréalisme de J. Chénieux-Gendron. Le Surréalisme d'H. Béhar et M. Carasson», no 53, juin 1985 , p. 8.

«Chère vieille France. Dictionnaire des littératures de langue française de J.-P. de Beaumarchais, D. Conty et A. Rey», no 54, septembre 1985 , p. 6.

«Éternelle ambiguïté de l'écrit et de la vie. La Douleur de M. Duras. Duras, Une lecture des fantasmes de M. Borgomano», no 55, octobre 1985, p. 16.

«la Salle de bain de J.-P. Toussaint», no 56, novembre 1985, p. 12.

«De la première à la dernière cigarette. Le Grand fumeur et sa passion d'O. Lesourne», no 57, décembre 1985, p. 12.

«Du hobby à la publication. Québécoises d'hier et d'aujourd'hui de. R. Prévost», no 58, février 1986, p. 14.

«Conversations avec le temps de D. Roche», no 58, février 1986, p. 13.

«Enfin de l'humour noir. Comment faire lamour avec un nègre sans se fatiguer de D. Laferrière», no 58 , février 1986, p. 6.

«Bien à vous... de ville Jacques-Cartier, P.Q. Les Lettres aux journaux de J. Ferron», no 59, mars 1986, p. 15. 
«Abus des mots et lecture biaisée. Éditorial», no 60, avril 1986, p. 2.

«Des avortures du langage. Entretien avec le Docteur André Roch Lecours et Michèle Neverts, no 60, avril 1986, p. 8-9.

«Harlequin: choral ou corral? Entretien avec Julia Bettinotti», no 62, été 1986, p. 16.

«Lentement, le féminin s'élabore. La Dépendance amoureuse l'indépendance amoureuse, Cahiers du Grif. Femmes, féminisme et recherche, Affer», no 62, été 1986, p. 22.

«la Littérature au crible de la psychanalyse et des femmes. Entretien avec Marcelle Marini», no 64, octobre 1986, p. 10.

«Si toi aussi tu m'abandonnes de P. Harvey et L. Vaillancourt», no 65, novembre 1986, p. 11.

«Une entreprise hardie. La Médée d'Euripide de M. Cardinal», no 67, février 1987, p. 20.

«l'Homme et l'originaire. Entretien avec Mikel Dufrenne», no 68, mars 1987, p. 14 .

\section{Divers}

"Quatre grandes artistes du Québec», Forces, no 27, 2e trimestre 1974, p. 44-48.

«Lectures», Vie des arts, vol. 22, no 90, printemps 1978, p. 79-83.

"Voyage autour d'une écriture», Revue de l'Université d'Ottawa, vol. 50, no 1, janvier-mars 1980 , p. 34-48.

«Messe en si», la Nouvelle Barre du jour, no 106, octobre 1981, p. 77-81.

«l'Émergence du féminin: la lumière diffuse et la subversion sourde: regards sur la littérature des années 70m, le Devoir, 21 novembre 1981, p. 13.

«Marguerite Duras, ni idole ni nouveau dieu», le Devoir, 19 décembre 1981, p. 32.

«Sanguine d'amour», la Nouvelle Barre du jour, no 116, septembre 1982, p. 69-74.

«Glossaire pour Nicole», la Nouvelle Barre du jour, colloque NBJ 1980, Traces, écriture de N. Brossard, nos 118-119, novembre 1982, p. 63-70.

«Féminisme et critique. L'autre lecture/Bibliographie complémentaire» (en collaboration avec Roger Chamberland), Québec français, no 47, octobre 1982, p. 34-35.

«Luce Irigaray: Passions d'une femme», le Devoir, ler mai 1982, p. 24. 
«Des parcours irréversibles: littérature québécoise et féminisme: le salon du livre de Montréal», le Devoir, 5 novembre 1983, p. 13.

«Sur l'odeur d'une idée», Possibles, vol. 8, no 3, 1984, p. 152-154.

«Le Mal d'écrire», la Nouvelle Barre du jour, no 140, juin 1986, p. 82-83.

«Petite Cantate à l'honnête voleuse», la Nouvelle Barre du jour, no 172, mars 1986, p. 85-89.

«Des essences volatiles aux fleurs de pissenlit», Critère, no 41, printemps 1986, p. 13-18.

«Sans lieu mais avec feu: Tessera», Cahiers de la femme, York University, vol. 7 , nos 1-2, printemps/été 1986, p. 231-232.

«Pourquoi Spirale?», Bulletin de l'Association des études canadiennes, vol. 8, no 2 , été 1986, p. 21.

«Au-delà de l'actualité. Chronique des revues», Voix \& images, vol. XII, no 1 (no 34), automne 1986, p. 161-164.

«De l'érudition: l'esprit et la lettre. Chronique des revues», Voix \& images, vol. XII, no 2 (no 35), hiver 1987, p. 342-346.

\section{COMMUNICATIONS ET PRODUCTIONS RADIOPHONIQUE/TÉLÉVISUELLE}

«la Femme, le Québec et les arts plastiques», Conférence canadienne des arts, mai 1975.

«Sur le surréalisme», Femmes d'aujourd'hui, Radio-Canada, 1980.

«Sur les écritures des femmes», Radio-Canada, 1981.

«la Critique au féminin dans le contexte canadien et québécois», colloque Dialogue, University York, Toronto, 1981.

«Quand des encres rouges dénudent le sens», Congrès de l'Association des professeurs de français du Québec, 1982.

«Subversion en rose, sur Yolande Villemaire», XXVe congrès de l'Association des professeurs de français des universités et collèges canadiens, Ottawa, 1982.

«Édition critique de l'oeuvre d'Hubert Aquin (genèse)», Acfas, 1982.

«Autour de Marguerite Duras», Actuelles, Radio-Canada, 1982.

«les Obscures clartés de la modemité», colloque de l'Académie canadiennefrançaise, Sainte-Adèle, 1983. 
«Je suis un noeud, sur France Théoret», Congrès de l'Association des professeurs de français des universités et collèges canadiens, Vancouver, 1983.

«D'un autre lieu commun», Opuscula Aesthetica Nostra, Edmonton, 1984.

«Des marques d'authenticité repérées au moi divisé jusqu'à l'insoutenable, dans Journal d'une femme soumise de Mara», Département d'études littéraires, UQAM, 1985.

«l'Honnête homme», Questions de notre temps, Radio-Canada, 1986.

«Des ambiguittés de la fiction à la tentation du récit», Congrès de l'APFUCC, Winnipeg, mai 1986.

«Ma mère et moi», En toutes lettres (réalisateur: André Major, lecteur: Gérard Poirier), Radio-Canada, 17 mars 1987.

«Radom», Souvenirs d'enfance et de jeunesse (rélisateur: André Major, lecteur: Françoise Faucher), Radio-Canada, 28 août 1987.

\section{ARTICLES SUR L'AUTEURE ET SON OEUVRE}

RENAUD, Jacques, «le Surréalisme et le Québec», le Devoir, 17 décembre 1977, p. 40.

JONES, Henri, «Suzanne Lamy. André Breton. Hermétisme et poésié dans Arcane 17», Livres et auteurs québécois, 1977, Québec, Presses de l'Université Laval, 1978, p. 249-252.

OUELLETTE-MICHALSKA, Madeleine, «Suzanne Lamy: du discours de maîtrise à la cadence des lunes", le Devoir, 2 février 1980, p. 21.

POISSANT, Louise, «Des femmes et des textes», Possibles, vol. 8, no 3, printemps 1984, p. 73-81.

ST-MARTIN, Lori, «la Critique au féminin», Spirale, no 43, mai 1984, p. 12.

THÉRY, Chantal, «Féminité, subversion, écriture», Lettres québécoises, no 34, été 1984, p. 68-69.

GAUVIN, Lise, «la Conscience des pièges de l'écriture féminine»,le Devoir, ler septembre 1984, p. 15.

LÉPINE, Stéphane, «la Maladie et la mort»,-le Devoir, 28 décembre 1985, p. 19.

MARTEL, Réginald, «la Beauté d'un texte parfait; Suzanne Lamy», la Presse, ler février 1986, p. E3.

BEAUDOIN, Réjean, «les Mouches du plafond», Liberté, no 165, juin 1986, p. 126-131.

NEPVEU, Pierre, «Tout l'horizon du vivant»; Spirale, no 71, été 1987, p. 2. 
80 VOIX \& IMAGES / 37, automne 1987

YANACOPOULO, Andrée, «la Trahison des corps», Spirale, no 71, été 1987, p. 8.

CORBEIL, Normand, «l'Amie Suzanne», Spirale, no 71, été 1987, p. 8-9.

COLLIN, Françoise, «Seeking Suzanne», Spirale, no 71, été 1987, p. 9.

THÉORET, France, «Résolument, le féminisme», Spirale, no 71, été 1987, p. 10.

Présences

MAILHOT, Laurent, MELANCON, Benoît, Essais québécois, Montréal, HMH, 1984 , p. $453-455$.

GAUDET, Gérald, Voix d'écrivains, entretiens, Montréal, Québec/Amérique, 1985, p. $235-245$. 